\title{
Design and implementation of a cognitive engine functional architecture
}

\author{
DONG Xu ${ }^{1,2^{*}}$, LI Ying ${ }^{2} \&$ WEI ShengQun ${ }^{2}$ \\ ${ }^{1}$ Institute of Communications Engineering, PLA University of Science and Technology, Nanjing 210007, China; \\ ${ }^{2}$ Institute of China Electronic System Engineering Corporation, Beijing 100141, China
}

Received October 13, 2011; accepted January 15, 2012; published online May 6, 2012

\begin{abstract}
Intelligence is the most important characteristic for cognitive wireless networks. A cognitive engine built on reconfigurable wireless networks is the key to implementing this characteristic. The design and implementation of a cognitive engine is important in research on the theory of initiative cognition for cognitive wireless networks. This paper first discusses research on cognitive loops, then investigates cognitive functions in the loop through the design of a universal cognitive engine functional architecture, and finally verifies the architecture on the platform of a cognitive engine prototype system.
\end{abstract}

cognitive radio, cognitive wireless network, cognitive loop, cognitive engine, prototype system

Citation: Dong X, Li Y, Wei S Q. Design and implementation of a cognitive engine functional architecture. Chin Sci Bull, 2012, 57: 3698-3704, doi: $10.1007 / \mathrm{s} 11434-012-5102-6$

"Cognition" is the scientific term for the process of thinking. Mitola et al. [1] introduced "cognition" to wireless communication and coined the phrase "Cognitive Radio" in 1999. From then on, cognitive radio and cognitive networks have attracted increased attention from researchers and research institutions [2-4], and have become a hotspot in the communication domain [5]. A cognitive network is able to sense information from the outside world and its internal state, and can then adjust the wireless network (including working frequency, air access, data protocols, and so on) by parsing and orienting the sensing information to adapt to changes in the environment. Moreover, a cognitive network can still learn to form new knowledge. Thus, a cognitive network is an intelligent network that can sense, make decisions, and learn like humans. Implementing such a wireless network requires a functional architecture as the brain to control the whole cognitive process, that is, a cognitive engine.

The cognitive engine integrates various cognitive functions (such as sensing, learning, and reasoning) so that it can intelligently control a wireless network to implement a cognitive cycle. Rieser [6], a researcher at Virginia Tech, first

*Corresponding author (email: dongxu1226@126.com) modeled the biologically inspired cognitive engine called BioCR. Later, his colleague Rondeau [7] further developed the theory of cognitive engines. He evolved cognitive engine modeling as a multi-objective optimization through genetic algorithms and built a cognitive engine architecture composed of a cognitive controller, sensors, a decision maker, optimizers, and interfaces. However, as this cognitive engine was purely designed for waveform optimization, it has limitations. In this paper, we set out to design a universal cognitive engine functional architecture by studying the cognitive loop.

\section{Cognitive loop}

A cognitive loop with feedback is a cognitive model that exists extensively in cognitive behavior. Take cognitive psychology for example. There are different cognitive loops for different memory models. The phonological loop in Baddeley et al. 's model [8] of working memory is a well known cognitive loop. In addition, cognitive loops are important in military decision making; the OODA (observe, orient, decide, and act) loop proposed by military strategist 
Boyd [9] has been widely studied and applied in military and commercial areas. Moreover, militarist Bryant [10] built the CECA (critique, explore, compare and adapt) loop based on the new principle of cognitive science for decision making in the context of command and control. When Mitola $[1,11]$ proposed the concept of cognitive radio, he defined a cognitive loop called OOPDAL (observe, orient, plan, decide, act, and learn) based on the OODA loop. The OOPDAL loop forms the basis for interaction between cognitive radio and the outside world and also for decision making. OOPDAL represents the necessary cognitive functions of cognitive radio and, compared to OODA, includes plan and learn functions. The CECA loop emphasizes goal-directed cognition and critical thinking; in other words, plan and learn. Therefore, OODA and CECA can verify the OOPDAL loop. Plan and learn functions are the key to distinguishing between cognitive radio and traditional radio.

Besides the OOPDAL loop, some cognitive radio researchers and research institutions have provided other cognitive loop models. Haykin [2] proposed a cognitive loop from the perspective of efficient spectrum usage. This cognitive loop contains three cognitive tasks: (a) radio-scene analysis, (b) channel identification, and (c) transmit-power control and dynamic spectrum management. These tasks interact with the radio environment to form the cognitive cycle. Researchers at Virginia Tech proposed a cognitive loop from the perspective of a physical-layer waveform adaption. This cognitive loop includes environment observation, scenario synthesis, case-based decision making, link configuration optimization, performance estimation, and reasoning functions. Each function interacts with the radio hardware to form the cognitive cycle [4]. Both the above cognitive loops were built for certain cognitive radio application scenarios. The functions of the two loops are related to the cognitive functions in the OOPDAL loop; in another words, the two loops can be decomposed into the OOPDAL loop or its subsets.

As shown in Figure 1, this paper adds a knowledge base to the OOPDAL loop, which clarifies obtaining and applying knowledge. The OOPDAL loop in Figure 1 is composed of an inner loop and an outer loop. The outer loop is the

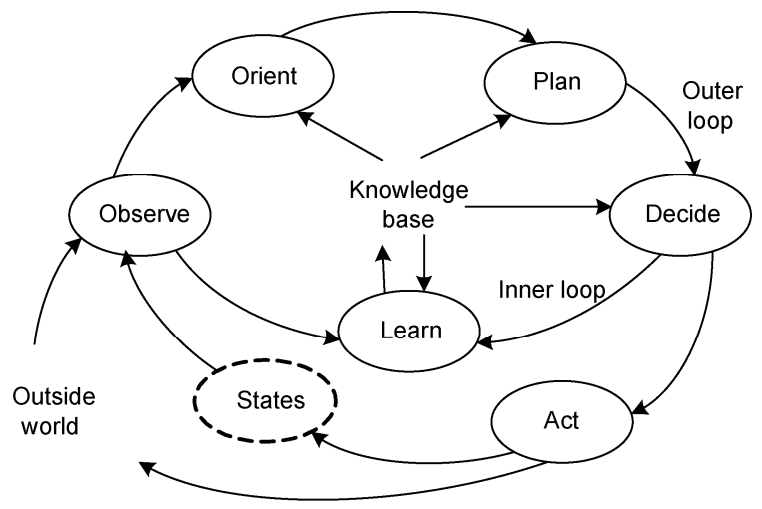

Figure 1 OOPDAL cognitive loop. decision loop, while the inner loop is the learning loop. The outer loop cooperates with the inner loop to implement the whole loop through the acquisition and application of knowledge. In the decision loop, the cognitive wireless network keeps "observing" information in the outside world and internal state. The observed information includes a wide variety of data coming from the wireless domain (link quality, interference temperature), network domain (load state, operating parameter, geographic location), user domain (user need, user experience), and policy domain (current spectrum policy). By modeling and parsing these data, the cognitive network can confirm its situation. "Orientation" represents data refining, which means removing information redundancy, extracting information contributing to decision making, and fusing related information to arrive at the original judgment by clearing, integrating and screening the sensing data. "Plan" produces one or more optimization objectives according to the user's need, current environment, and self state. "Decision" making identifies optimization objectives and executes appropriate algorithms based on the orientation. "Action" implements the result of the decision and changes the outside world and internal state. These changes are observed again on entering the next cycle. In the learning loop, the cognitive wireless network obtains knowledge from historical experiences of the outer loop and stores the knowledge in the knowledge base to guide the execution of the decision loop.

In the cognitive cycle model, both "plan" and "learn" are key steps. "Plan" reflects that the cognitive wireless network is not limited to a single task, but dynamically produces pointed optimization objectives in accordance with different tasks and requirements; in other words, the cognitive wireless network is goal-directed. "Learn" enables the cognitive wireless network to intelligently remember the knowledge obtained from historical experiences, and thus, it is the most important element of intelligence in a cognitive wireless network. It can be said that "plan" and "learn" are two marked characteristics for distinguishing cognitive wireless network from other networks.

The introduction to cognitive loops given above shows that the implementation of a complex loop needs to deal with difficulties, covering several domains, regarding the methods and technologies of cognitive wireless networks. If all the difficulties in the implementation of each cognitive loop's function could be solved according to the user's wishes, we would have created a cognitive engine. A cognitive engine is essentially an integration of methods and technologies for implementing functions of a cognitive loop.

\section{Design of a cognitive engine functional architecture}

Cognitive engine technologies have been intensively researched at Virginia Tech, where artificial intelligence 
technologies such as neural networks, case-based reasoning and genetic algorithms have been applied successfully in building cognitive engines [12]. Besides this, cognitive engines based on vague information of channel states and environments, a multi-antenna system cognitive engine, and the robust training problem in cognitive engines have been widely researched. Nevertheless, these studies were all aimed at specific applications with most related to link adaption and wireless resource management. This paper aims to build a general cognitive engine and thus, a functional architecture for a more universal cognitive engine needs to be designed.

\subsection{Supporting technologies}

The cognitive engine is an intelligent system integrating observing, decision making, learning and other functions. Implementation of so many functions requires a variety of related supporting technologies. Moreover, the different technologies need to be fused to construct a flexible and scalable cognitive engine functional architecture.

A cognitive network first needs observations for cognition [1]. The capability of observing is a precondition of executing a cognitive engine, so observation technology is the main problem for research on cognitive radio. Observation technologies can be divided into two application categories, namely, obtaining information and representing knowledge.

Obtaining information includes spectrum sensing, link quality measurement, location and timing. Spectrum sensing is the key to applying cognitive radio technology in efficient spectrum usage, and thus, it has been widely studied. Traditional spectrum sensing methods include matched filter detection, energy detection, and cyclostationary feature detection [13]. Each of these methods has its advantages and disadvantages. Besides these, cooperative detection is gaining more and more attention.

A cognitive wireless network needs not only to obtain information, but also to transform the information into machine-readable structures in natural language or another medium. The wireless network knows what the information means, and this is known as knowledge representation. Knowledge representation is included in knowledge construction, which means knowledge modeling. There are several approaches to constructing knowledge, such as ontologies, production rules and so on. Knowledge representation is so important that Mitola proposed a radio knowledge representation language (RKRL) as the foundation of his cognitive radio sensing, learning, and reasoning.

Artificial intelligence is another key technology for implementing functions of cognitive engines such as reasoning, decision making and learning. Artificial intelligence already has a mature theoretical basis [14], but application of artificial intelligence to cognitive radio is still in its infancy.

Expert systems have been used successfully in artificial intelligence [15]. An expert system, comprising a know- ledge base and a reasoner, is a computer system for simulating an expert's decisions. It can be used in cognitive wireless network reasoning processes for orientation and decision [16].

Case-based reasoning as an artificial intelligence technology involves obtaining solutions for new issues based on past experiences and cases. Thus, case-based reasoning can also be used in cognitive engine inference $[4,12]$. The difference between case-based reasoning and expert systems is that the former is based on experiences, whereas the latter is based on a priori knowledge.

Genetic algorithms apply biological principles of organic evolution and hereditism to optimize objectives. The principle of genetic algorithms is finding a set of parameters (genes) that maximizes the objective function. Genetic algorithms can implement the decision function in a cognitive engine $[7,16]$. By defining various radio parameters as genes on a chromosome, the cognitive engine can optimize parameters to obtain a set of optimal parameters to determine the system reconfiguration.

Neural networks are made up of interconnecting artificial neurons that mimic the properties of biological neurons. Such a network can be used for solving artificial intelligence problems, e.g., machine learning. A neural network can be used in spectrum sensing, radio parameter adaptive decision and adjustment, and prediction of wireless network performance metrics such as bit error rate, throughput, delay, and so on [17-20].

Besides these supporting technologies of cognitive engines, interface and protocol design is also necessary for implementing a cognitive engine, although this is not discussed here.

\subsection{Functional architecture}

Constructing a cognitive engine requires not only the supporting technologies mentioned above, but also the design of a functional architecture for integrating these technologies and implementing the cognitive loop. Virginia Tech's cognitive engine [4] was designed purely for adapting physical and link layers. Such a cognitive engine implements the cognitive loop from environmental awareness, through decision making, to waveform parameter optimization. This paper however, assumes that the cognitive engine is not designed purely for certain wireless tasks or wireless network layers. The architecture of the cognitive engine should be fundamental, versatile and general for various application scenarios. It should not only integrate the different kinds of technologies for achieving different cognitive functions, but also be flexible and scalable for conveniently combining, invoking and adding various technologies. Thus a universal cognitive engine functional architecture has been designed, as shown in Figure 2.

The universal cognitive engine is composed of a cognitive core, scheduler, user interface, sensor interface, and 


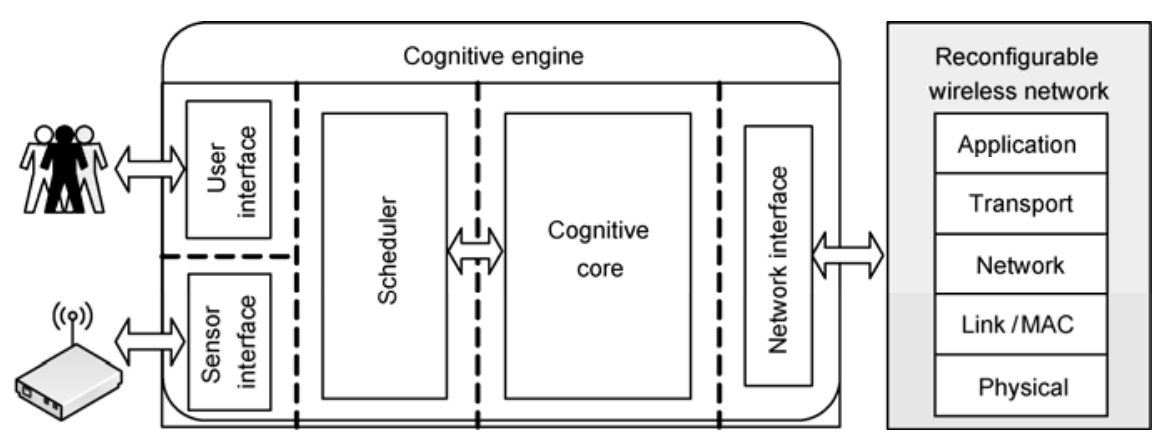

Figure 2 Universal cognitive engine functional architecture.

network interface. The cognitive core integrates various artificial intelligence technologies; the scheduler invokes and combines different technologies to implement cognitive functions and complete certain cognitive tasks in particular surroundings; the user interface provides information about the user domain such as the user's needs and experiences; the sensor interface collects information about the wireless domain like channel state; and the network interface obtains information about the network domain such as network performance. It should be noted that information about the policy domain should be gathered by the policy engine, which can exchange information with the cognitive engine.

The cognitive core is the kernel of the cognitive engine. The structure of the cognitive core, illustrated in Figure 3, includes a data base, learner, reasoner, and optimizer. Each component includes some kind of artificial intelligence technology and data base system. The cognitive engine can be updated by adding new technologies or tools to the cognitive core. In the cognitive engine execution process, the scheduler invokes related components of the cognitive core according to the different functional requirements in the cognitive loop. For example, when the cognitive engine obtains sensing information, the scheduler temporarily stores the information in a data base in the cognitive core. Then the reasoner is invoked for decision making and the optimizer is called by the scheduler to optimize the parameters. Finally, the scheduler invokes the learner to train new cases to form new knowledge. This completes the cognitive cycle. No step in the cognitive cycle can be implemented

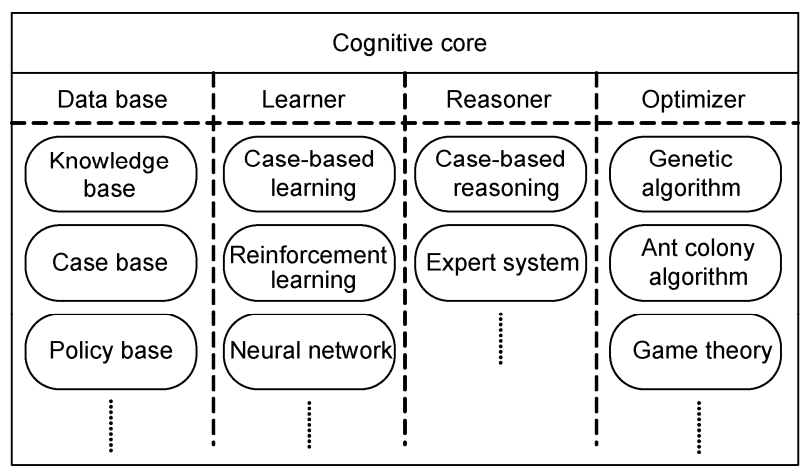

Figure 3 Structure of cognitive core. without invoking the related function in the cognitive core. Therefore, it is obvious that the cognitive core is most important for the cognitive engine.

From the design of the functional architecture for the cognitive engine given above, the cognitive engine has the following characteristics: (a) plenty of supporting technologies; (b) flexibility in the sense of scheduling different technologies to complete various tasks; and (c) scalability by conveniently joining new technologies.

\section{Verification of the cognitive engine prototype system}

Based on the functional architecture, we have developed a cognitive engine prototype system, which is verified on the experimental platform.

\subsection{System structure}

As shown in Figure 4, the structure of the proposed cognitive engine prototype system comprises three parts: cognitive engine, reconfigurable radio platform, and wireless environment.

The wireless environment is generated by a vector signal generator (VSG) and a connected PC. Matlab simulation software in the PC controls the VSG to generate a related waveform to mimic changes in the wireless environment such as the appearance of primary users and interferences.

The reconfigurable radio platform is a video wireless communication system, which includes two series of software radios and connected video equipment deployed separately in the sender and receiver. Software radio is composed of the universal software radio peripheral (USRP) and assorted GUN Radio software toolkit. USRP and GUN Radio can be used to reconfigure the software waveform by adjusting various parameters in the physical and link layers.

The cognitive engine comprises a PC and a sensor. The sensor is also a USRP for observing the wireless environment. The PC integrates all cognitive function models to implement the above design of a universal cognitive engine functional architecture, as illustrated in Figure 5. 


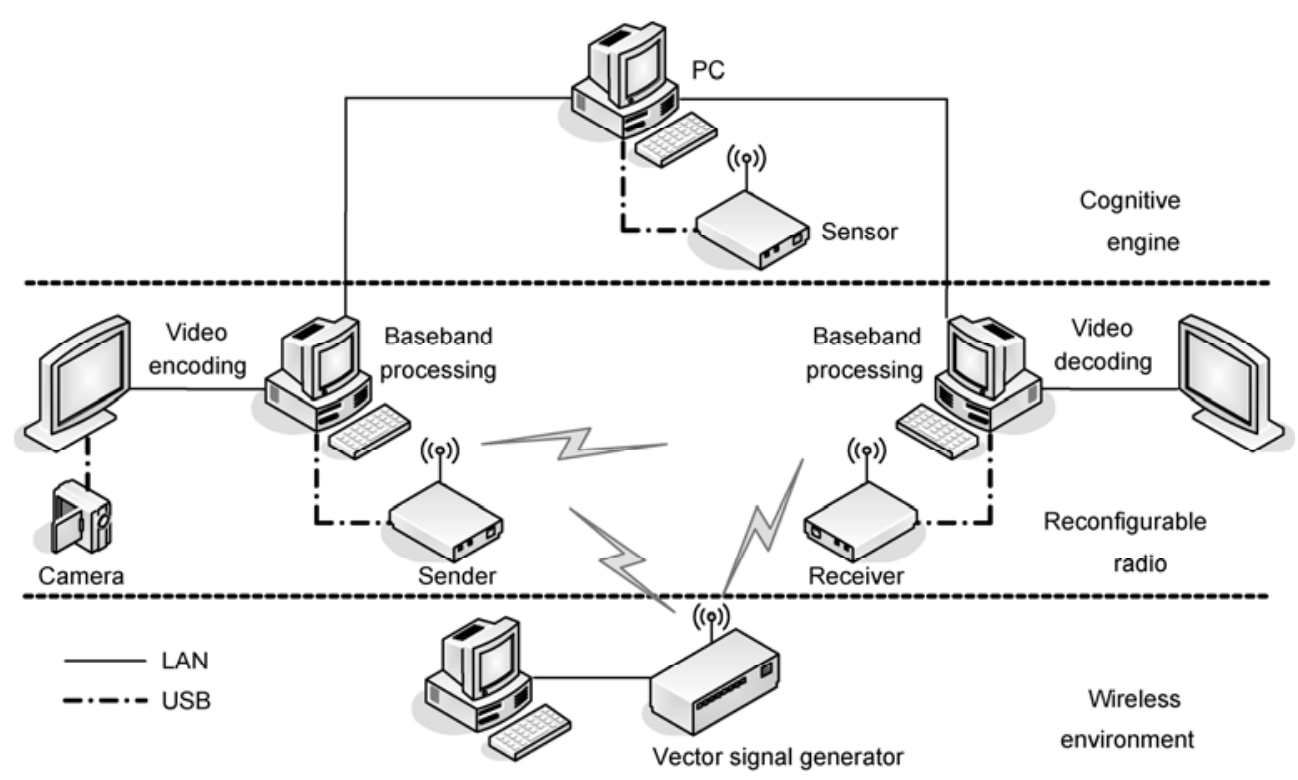

Figure 4 Structure of cognitive engine prototype system.

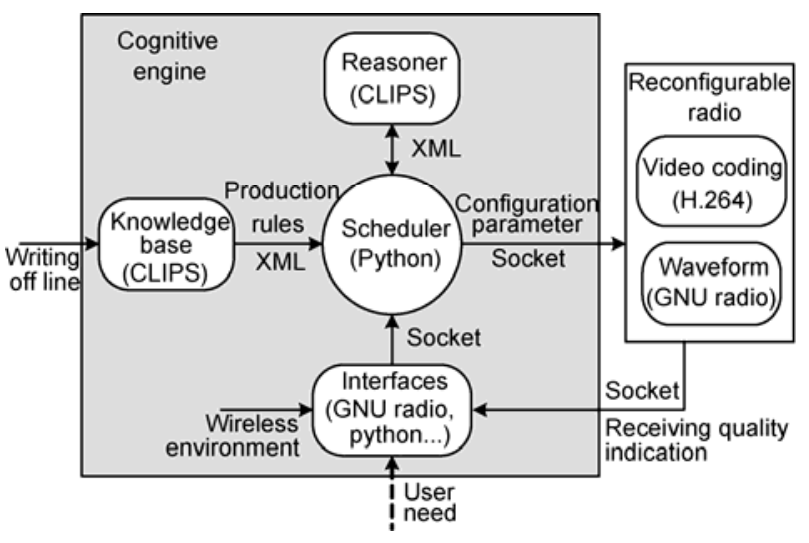

Figure 5 Cognitive engine architecture in prototype system.

The cognitive engine architecture contains a scheduler, reasoner, knowledge base, and different interfaces. The scheduler is written in Python and controls the entire cognitive cycle. The reasoner and knowledge base are implemented as CLIPS expert systems and interact with the scheduler through XML. In CLIPS, knowledge is represented as production rules, which can be stored in an offline knowledge base. When decision making is needed, the scheduler invokes the related knowledge for reasoning and sends the parameters obtained by the reasoning to the reconfigurable radio platform through socket messages. Different interfaces are used for collecting information about wireless, network, and user domains. The information is sent to the scheduler to trigger the cognitive cycle.

\subsection{Functions verification}

Each function of the cognitive engine functional architecture has been tested on the prototype system in a dynamic spectrum access scenario to verify the feasibility of the architecture.

In the dynamic spectrum access scenario, there are several channels in the prototype system. The VSG randomly generates different waveforms in different channels, which represent appearances of primary users. The video communication system is the secondary user that senses the state of each channel and chooses an idle channel for the communication by adjusting the waveform parameters. Detailed parameters are given in Table 1, while the transmission rates and bandwidths for different modulation modes are given in Table 2.

First, the cognitive engine observes information about channel states and stores the information in fact lists. A fact includes the channel number, busy/idle state, and idle bandwidth. " 1 " denotes that the channel is busy, while " 0 " denotes that the channel is idle. The idle bandwidth is quantized for five levels $(0,250,500,800$, and $1100 \mathrm{kHz})$,

Table 1 Waveform parameters

\begin{tabular}{cc}
\hline Adjustable parameter & Parameter range \\
\hline Carrier frequency & $2300-2900 \mathrm{MHz}$, step $1 \mathrm{~Hz}$ \\
Emission power & $0-50 \mathrm{~mW}$ \\
Modulation mode & DBPSK, DQPSK, GMSK \\
Channel coding & $1,1 / 2,1 / 3,2 / 3$ convolution coding \\
Frame length & $267-1602$ bytes, step 267 bytes \\
\hline
\end{tabular}

Table 2 Transmission rates and bandwidths for different modulation modes

\begin{tabular}{cll}
\hline Modulation & Transmission rate & Bandwidth range \\
\hline GMSK & $125 \mathrm{k}-4 \mathrm{Mbps}$ & $125 \mathrm{k}-4 \mathrm{MHz}$ \\
DBPSK & $125 \mathrm{k}-2.666 \mathrm{Mbps}$ & $125 \mathrm{k}-2.7 \mathrm{MHz}$ \\
DQPSK & $250 \mathrm{k}-5.333 \mathrm{Mbps}$ & $125 \mathrm{k}-2.7 \mathrm{MHz}$ \\
\hline
\end{tabular}




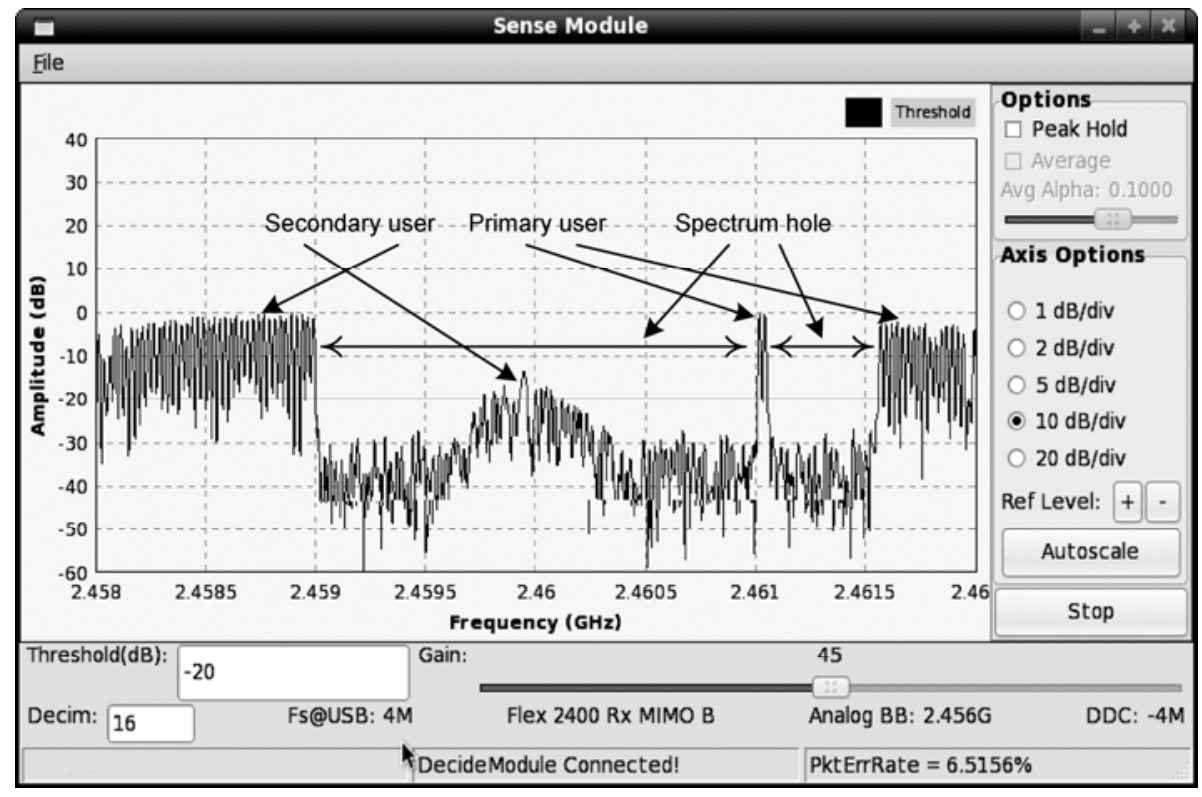

Figure 6 Verification of functions.

which are numbered from 0 to 4 .

Second, the cognitive engine invokes the reasoner in the cognitive core to reason based on the facts and knowledge in the knowledge base. The knowledge is represented as production rules, which are composed of preconditions and postconditions. The precondition determines the facts while the postcondition produces the actions associated with the judgment. The knowledge about communication in an idle channel can be expressed as follows. If channel $x$ busy/idle state $=0$, then communication channel number $=x$. Thus, $\mathrm{a}$ video communication system can avoid the primary user and transmit videos on an idle channel to improve spectrum efficiency.

Third, if there are different idle channels, the cognitive engine invokes the reasoner to choose the maximum idle bandwidth based on the idle bandwidths and related rules in the knowledge base. Thus, the system can increase the communication bandwidth and transmission rate.

Finally, the cognitive engine can adjust the coding rate, modulation mode, and frame length parameters according to the sensing information regarding channel states and users' needs. The users' needs can be satisfied and goal-directed initiative cognition implemented.

As shown in Figure 6, the above dynamic spectrum access scenario tests the capabilities of the cognitive engine, such as sensing, decision making, action, and application of knowledge. This verifies the feasibility of the cognitive engine functional architecture.

\section{Discussion}

Research on a cognitive engine functional architecture is most important for implementing cognitive wireless net- works. This paper presented a study of cognitive loops and the construction of a cognitive loop model. Then, from the implementation of the loop perspective, the universal cognitive engine functional architecture was designed by analyzing the cognitive engine support technologies. Finally we verified the feasibility of the architecture for building a cognitive engine prototype system. This research is still in the development stage; future work will keep improving and evolving the cognitive engine functional architecture. By the way, research on a cognitive engine plan and learning is not enough at present. Implementation of planning and learning is fundamental to real intelligence. Therefore this domain should be studied intensively. The design of a practical architecture, interfaces, and protocols for cognitive wireless network is concerned with whether cognition can flexibly and effectively converge heterogeneous networks, and therefore, this is also fundamental to the study.

This work was supported by the National Basic Research Program of China (2009CB320403), the National Natural Science Foundation of China (60832006, 60832008), and the National Science and Technology Major Project of China (2009ZX03007-004).

1 Mitola J, Maguire G Q. Cognitive radio: Making software radios more personal. IEEE Pers Commun, 1999, 6: 13-18

2 Haykin S. Cognitive radio: Brain-empowered wireless communications. IEEE J Sel Area Comm, 2005, 23: 201-220

3 Federal Communications Commission. Spectrum policy task force report, ET Docket No. 02-135. Washington DC: FCC, 2002

4 MacKenzie A B, Athanas P, Buehrer R M, et al. Cognitive radio and networking research at Virginia Tech. P IEEE, 2009, 97: 660-688

5 Fortuna C, Mohorcic M. Trends in the development of communication networks: Cognitive networks. Comput Netw, 2009, 5: 13541376

6 Rieser C J. Biologically inspired cognitive radio engine model utilizing distributed genetic algorithms for secure and robust wireless 
communications and networking. Doctorial Dissertation. Virginia: Virginia Tech, 2004

7 Rondeau T W. Application of artificial intelligence to wireless communication. Doctorial Dissertation. Virginia: Virginia Tech, 2007

8 Baddeley A, Sala S D. Working memory and executive control. Philos T R Soc B, 1996, 351: 1397-1404

9 Fusano A, Sato H, Namatame A. Study of multi-agent based combat simulation for grouped OODA loop. In: Proceedings of the SICE Annual Conference, 2011, Tokyo. Washington DC: IEEE, 2011. 131-136

10 Bryant D J. Critique, explore, compare, and adapt (CECA): A New Model for Command Decision Making, TR 2003-105. Toronto: Defense R\&D Canada, 2003

11 Mitola J. Cognitive radio: An integrated agent architecture for software defined radio. Doctorial Dissertation. Stockholm: Royal Institute of Technology, 2000

12 He A, Bae K K, Newman T R, et al. A survey of artificial intelligence for cognitive radios. IEEE T Veh Technol, 2010, 59: 1578-1592

13 Cabric D, Mishra S M, Brodersen R W. Implementation issues in spectrum sensing for cognitive radios. In: Proceedings of 38th Asilomar Conference on Signals, Systems and Computers, 2004, California. Washington DC: IEEE, 2004. 772-776

14 Luger G F. Artificial Intelligence: Structures and Strategies for Com- plex Problem Solving. 5th ed. Edinburgh: Pearson Education Limited, 2005

15 Giarratano J C, Riley G D. Expert System: Principles and Programming. 4th ed. Stamford: Thomson learning, 2006

16 Newman T R. Multiple objective fitness functions for cognitive radio adaptation. Doctorial Dissertation. Kansas: University of Kansas, 2008

17 Baldo N, Tamma B R, Manoj B S, et al. A neural network based cognitive controller for dynamic channel selection. In: Proceedings of the IEEE International Conference on Communications, 2009, Dresden. Washington DC: IEEE, 2009. 1-5

18 Zhu X, Liu Y, Weng W, et al. Channel sensing algorithm based on neural network for cognitive wireless mesh network. In: Proceedings of the IEEE International Conference on Wireless Communications, Networking and Mobile Computing, 2008, Dalian. Washington DC: IEEE, 2008. 1-4

19 Tumuluru V K, Wang P, Niyato D. A neural network based spectrum prediction for cognitive radio. In: Proceedings of the IEEE International Conference on Communications, 2010, Cape Town, South Africa. Washington DC: IEEE, 2010. 1-5

20 Baldo N, Zorzi M. Learning and adaptation in cognitive radios using neural networks. In: Proceedings of the IEEE Consumer Communications and Networking Conference, 2008, Las Vegas. Washington DC: IEEE, 2008. 998-1003

Open Access This article is distributed under the terms of the Creative Commons Attribution License which permits any use, distribution, and reproduction in any medium, provided the original author(s) and source are credited. 